\title{
Probabilistic finite element analysis of fatigue life of additively manufactured clasp
}

\author{
Kento ODAKA ${ }^{1}$, Naoki TAKANO ${ }^{2}$, Hideo TAKIZAWA ${ }^{3}$ and Satoru MATSUNAGA ${ }^{4}$ \\ ${ }^{1}$ Department of Oral and Maxillofacial Radiology, Tokyo Dental College, 2-9-18 Kandamisaki-cho, Chiyoda-ku, Tokyo 101-0061, Japan \\ ${ }^{2}$ Department of Mechanical Engineering, Keio University, 4-1-1 Hiyoshi, Kohoku-ku, Yokohama, Kanagawa 223-8521, Japan \\ ${ }^{3}$ Mechanical Engineering Department, Nippon Institute of Technology, 4-1 Gakuendai, Miyashiro-machi, Minamisaitama-gun, Saitama 345-0826, \\ Japan \\ ${ }^{4}$ Department of Anatomy, Tokyo Dental College, 2-9-18 Kandamisaki-cho, Chiyoda-ku, Tokyo 101-0061, Japan \\ Corresponding author, Satoru MATSUNAGA; E-mail: matsuna@tdc.ac.jp
}

The present study was aimed to develop a probabilistic finite element method (FEM) that predicts the variability in the fatigue life of additively manufactured clasp so that it can be used as a virtual test in the design phase before manufacturing. Titanium alloy (Ti$6 \mathrm{Al}-4 \mathrm{~V}$ ) clasp with integrated chucking part, which was designed for experimental fatigue test to validate the computational method, was investigated. To predict the lower bound, an initial spherical defect was assumed in the region where stress concentration was predicted. The Smith-Watson-Topper (SWT) method, Bäumel \& Seeger rule, elasto-plastic FEM, and zooming FEM were used. The influence of assumed initial defect on the fatigue life was significant, and the large variability in the fatigue life was predicted. This study demonstrated that the proposed practical computational method can simulate the large variability in the fatigue life of titanium alloy clasp, which is useful in its design before manufacturing.

Keywords: Finite element method, Clasp, Additive manufacturing, Fatigue life

\section{INTRODUCTION}

Additive manufacturing (AM) of metallic and polymeric products will become one of the major manufacturing methods together with many conventional ones such as casting or milling in dental field ${ }^{1-4)}$. Culmone et al.5) wrote that AM is opening the door to a new approach in the production of medical devices, which allows the complexity of their designs to be pushed to the extreme. Together with the collections of design principles based on the clinical experiences ${ }^{6}$, a numerical approach using finite element method (FEM) is essential in the use of AM. There are two directions at this moment in the use of FEM for AM. They are the manufacturing process simulation ${ }^{7}$ and the topology optimization ${ }^{8,9}$. Some sort of numerically designed structures ${ }^{10-12)}$ can be manufactured only by AM. With this background, this paper newly proposes a probabilistic finite element analysis (FEA) procedure to perform the virtual test for quality assurance of additively manufactured clasp by predicting the largely scattered fatigue life.

One drawback of AM lies in the higher possibility of variety of defects in the product ${ }^{13,14}$, which governs the failure behaviors ${ }^{15)}$. Snow et al. ${ }^{14)}$ summarized the current understanding of formation mechanisms for gas porosity, lack-of-fusion and flaws related to melt pool instabilities, and described their impact on mechanical properties including fatigue life showing large scattering. Douellow et al. ${ }^{16)}$ proposed a statistical model to identify the fatigue limit, and wrote that it leads to the rapid

Color figures can be viewed in the online issue, which is available at J-STAGE.

Received Jun 21, 2021: Accepted Oct 27, 2021

doi:10.4012/dmj.2021-174 JOI JST.JSTAGE/dmj/2021-174 optimization of AM process with respect to the fatigue performance.

Needless to say, the fatigue behavior is important in the design and evaluation of clasp ${ }^{17)}$ and therefore experimental studies have been carried out ${ }^{18)}$. Behr et al. $\left.{ }^{19}\right)$ investigated the 10-years survival rate of clasp-retained removal partial dentures and revealed that fracture was observed in clasps for $16.1 \%$ of patients, which was almost three times higher than other parts such as connectors. In the study of new materials for clasp ${ }^{20}$, the cantilever type bending fatigue test was carried out. Comparison of deformation and retentive force under cyclic load between cobalt chromium alloy and aryl ketone polymer was studied experimentally ${ }^{21}$. However, the numerical prediction of fatigue life is still a challenging research theme, because the fracture initiation site may include microscopic features and surface roughness features together with the above-mentioned variety of defects, and they are regarded as uncertainty factors in FEA. To consider the influence of uncertainty factors, a sort of probabilistic simulation technique ${ }^{22)}$ is needed such as the Monte Carlo simulation, but it usually requires huge computational cost. This study presents a practical probabilistic FEA by predicting only the lower bound of the fatigue life by assuming a small defect as a most influential uncertainty factor.

Ghosh et $a .^{23)}$ studied on the uncertainty analysis and wrote that quality control in AM can be achieved through variation control of the quantity of interest. Moussa et $a l .{ }^{24)}$ emphasized the importance of including process-induced geometric defects during the design process. In this study, following the experimental 
works ${ }^{15,25)}$, an initial spherical defect was assumed in the FEA to simulate the large variability in fatigue life of clasp, and especially to predict the lower bound. As another uncertainty factor, the variability in the material properties was investigated in the same way to predict the bounds.

The aim of present study was to develop a probabilistic FEM that predicts the variability in the fatigue life of additively manufactured clasp. The main feature of this study is to predict the scattering range of the fatigue life of additively manufactured Ti-6Al-4V clasp by analyzing the stress/strain without and with assumed initial spherical defect.

\section{MATERIALS AND METHODS}

Overview of fatigue life prediction method

The fatigue life $N_{f}$ was calculated by the Smith-WatsonTopper (SWT) method ${ }^{26)}$ in Eq. (1), where $E$ is the Young's modulus, $\Delta \varepsilon$ is the repeatedly applied strain and $\sigma_{\max }$ is the maximum stress. The calculated maximum value of the maximum principal strain and maximum principal stress by FEA were used for $\Delta \varepsilon$ and $\sigma_{\max }$ in this study. Note that only static FEA is required for the fatigue life prediction by the SWT method.

$$
\frac{\Delta \varepsilon}{2} \sigma_{\max }=\frac{\left(\sigma_{f}^{\prime}\right)^{2}}{E}\left(N_{f}\right)^{2 b}+{\sigma_{f}^{\prime} \varepsilon_{f}^{\prime}}_{f}\left(N_{f}\right)^{b+c}
$$

Other parameters are defined by the Bäumel \& Seeger rule $^{27)}$ as in the followings.

$$
b=-0.095, c=-0.69, \varepsilon_{f}^{\prime}=0.35, \sigma_{f}^{\prime}=1.67 \sigma_{u}
$$

where $\sigma_{u}$ is the ultimate strength.

The SWT method is advantageous over other prediction methods in the point that the effect of stress ratio can be considered ${ }^{28)}$. The parameters used in the SWT method was determined by the Bäumel \& Seeger rule after the static tensile test of dumbbell type specimen made by AM. This rule is applicable to titanium alloy and aluminum alloy. Hence, in this study, titanium alloy (Ti-6Al-4V) is used, instead of cobalt chromium alloy.

\section{Additively manufactured clasp model}

Figure 1(a) shows the targeted clasp model manufactured by selective laser melting (SLM) technique using EOS M290 printer (EOS, Munich, Germany) with the help of J-3D. (Nagoya, Japan). Titanium alloy (Ti6Al-4V) supplied by EOS, was used as mentioned above. The support structure and the building direction with 30 $\mu \mathrm{m}$ layer pitch are shown in Fig. 1(b).

Together with FEA, experimental fatigue test was carried out to compare the fatigue lives between FEA and experiment using a small-stroke fatigue testing machine IMC-0608 (Imoto Machinery, Kyoto, Japan) in Fig. 2. The fatigue test was performed under displacement control, and the reaction force was measured. The fatigue fracture was identified by the $25 \%$ reduction of the reaction force according to JIS Z 2283. The fatigue (a)

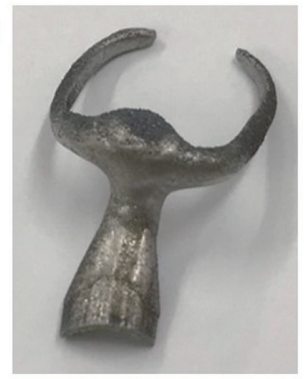

(c)

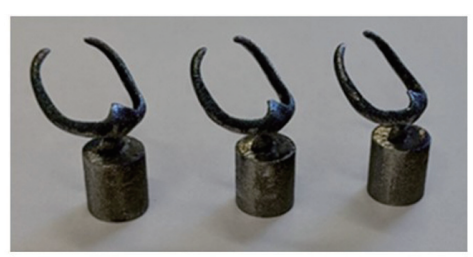

Building

(b) direction

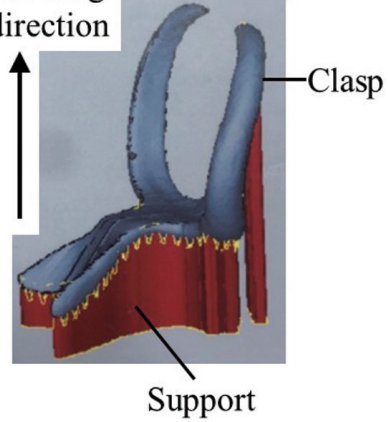

(d)
Building direction

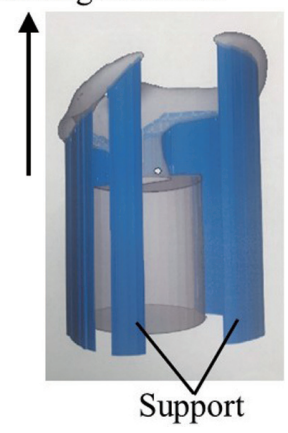

Fig. 1 Geometry model of typical clasp used in this study and support structure for additive manufacturing. (a) Additively manufactured clasp with (b) support structure. (c) Fatigue test specimen with cylindrical chucking part. (d) Support structure to additively manufacture the specimen in (c).

(a)

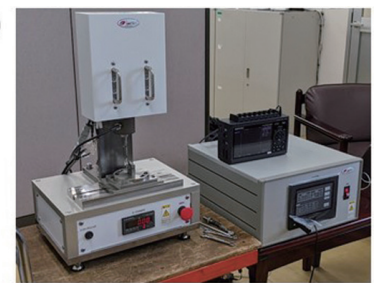

(b)

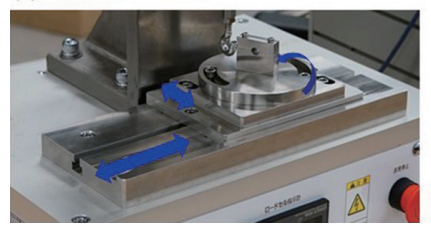

(c)

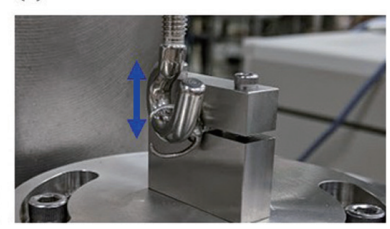

Fig. 2 (a) Fatigue test apparatus designed for clasp specimen with cylindrical chucking part, (b) magnified view of chucking jig with 3 d.o.f. alignment shown in blue arrows, and (c) loading device to apply prescribed cyclic displacement in blue arrows.

test finished when the specimen was broken or the fatigue life exceeded $1.5 \times 10^{5}$ cycles. The machine allows chucking part, which includes in-plane 2-dimensional 
translational movements and 1 rotational movement, as indicated by blue arrows in Fig. 2(b). The magnified view of the chucking part and the loading device are in Fig. 2(c). The cyclic stroke of $0 \mathrm{~mm}$ and approximately $0.25 \mathrm{~mm}$ was applied to the specimen in the experiment. Note that, in the numerical analyses that solved static elasto-plastic problem, the displacements were applied up to $0.5 \mathrm{~mm}$.

To compare the fatigue life between numerical prediction and experimental result, three specimens of a clasp model with cylindrical chucking part in Fig. 1(c) were manufactured and tested. To assure the geometry of the cylinder shape in the chucking part for the jig in Fig. 2(c), the building direction in Fig. 1(d) was employed. In this case, the support structure was needed in the arm part of the clasp, which is not desirable because removing the sacrificed support may cause problem in the arm part.

\section{Material properties}

Static tensile test was carried out for the dumbbell type specimen as shown in Fig. 3(a). This is the half size of type-5 specimen defined in JIS Z 2241(2011). The same printer and titanium alloy powder (EOS) as well as the printing service company (J-3D) were used for the dumbbell type specimen. Five specimens were additively manufactured with no support structure. Shimadzu Autograph AG-100kN and strain gage KYOWA KFGS-2120-C1-16 were used. Figure 3(b) shows the relationship between true stress and plastic strain, where the maximum response curve, minimum response curve and the averaged curve obtained by 5 specimens are plotted. The 5 specimens showed relatively large variability depending on the position on the powder bed during AM.

For FEA, the Swift hardening law in Eq. (3) was adopted, where $\bar{\sigma}$ and $\bar{\varepsilon}^{p}$ are Mises stress and equivalent plastic strain. $C, \alpha$ and $n$ are the coefficients.

$$
\bar{\sigma}=C\left(\alpha+\bar{\varepsilon}^{p}\right)^{n}
$$

Since the static tensile test results are used in the following uncertainty modeling, the obtained Young's modulus, yield stress, ultimate strength and the coefficients in the Swift law are summarized in this section as shown in Table 1 . The average material model is firstly used in FEA, but the influence of the variability in the properties is discussed later. Also, the comparison with the catalogue data offered by EOS, is described in the discussion.

\section{Uncertainty modeling}

This study simulated the fatigue test as shown in Figs. 1(c), (d) and 2. For validating the numerical model, the uncertainty involved in the geometry, material
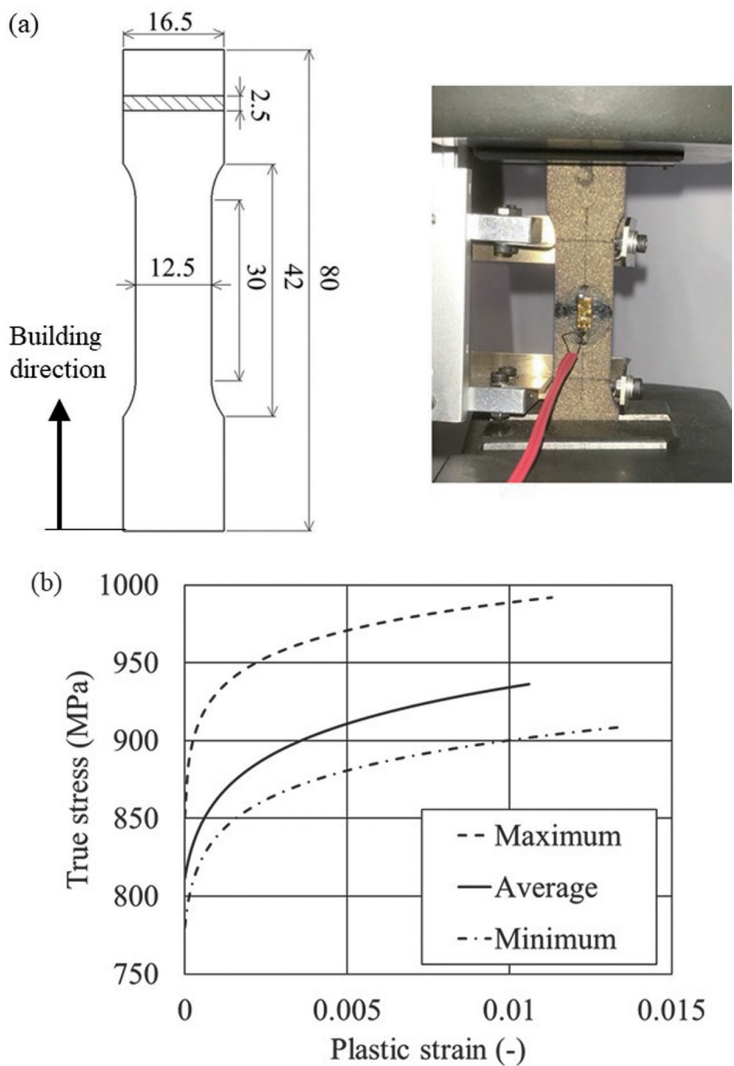

Fig. 3 Experimental work to obtain the material properties of additively manufactured titanium alloy (Ti-6Al-4V).

(a) Dumbbell type static test specimen. The right figure shows the experimental setup. (b) Relations between true stress and equivalent plastic strain obtained by static tensile tests for 5 specimens. The variability was observed between maximum and minimum curves.

Table 1 Material properties of additively manufactured titanium alloy (Ti-6Al-4V) obtained by static tensile test of dumbbell type specimen in this study

\begin{tabular}{|c|c|c|c|c|c|c|}
\hline \multirow{2}{*}{ Models } & \multirow{2}{*}{$\begin{array}{l}\text { Young's } \\
\text { modulus } \\
E(\mathrm{GPa})\end{array}$} & \multirow{2}{*}{$\begin{array}{c}\text { Yield } \\
\text { stress } \\
\sigma_{Y}(\mathrm{MPa})\end{array}$} & \multirow{2}{*}{$\begin{array}{c}\text { Ultimate } \\
\text { stress } \\
\sigma_{u}(\mathrm{MPa})\end{array}$} & \multicolumn{3}{|c|}{ Parameters for Swift hardening law } \\
\hline & & & & $C(\mathrm{MPa})$ & $\alpha$ & $n$ \\
\hline Maximum & 116.2 & 851 & 992 & 1,117 & $0.34 \times 10^{-4}$ & 0.027 \\
\hline Average & 109.8 & 813 & 936 & 1,111 & $2.50 \times 10^{-4}$ & 0.038 \\
\hline Minimum & 101.2 & 780 & 909 & 1,043 & $1.15 \times 10^{-4}$ & 0.032 \\
\hline
\end{tabular}


properties, constraint condition, loading condition and initial condition should be considered. Since the authors ${ }^{2}$ have shown the geometrical accuracy, the uncertainty in geometry was ignored in this study. The variability in material properties in Fig. 3(b) was considered as maximum, average and minimum material models. The differences among three models are discussed through both static FEA and predicted fatigue life. The uncertainty in the constraint condition was discussed experimentally later. The uncertainty in the loading direction was ignored by carefully setting the specimen using the specially designed jig in Fig. 2(b). The uncertainty in the initial condition is described in the next section.

\section{Determination of diameter of spherical defect and zooming FEM}

As the uncertainty in the initial condition, the spherical defect is assumed to predict the lower bound of the fatigue life at the location where stress concentration occurs. To determine the dimension of the assumed defect, a demonstrative example of S-shaped specimen was analyzed first. The influence of the diameter of the spherical defect was investigated for a demonstrative example of S-shaped component as shown in Fig. 4(a). Also, the constraint condition and applied prescribed displacement are shown. Three diameters ranging from $1 \mu \mathrm{m}$ to $200 \mu \mathrm{m}$ were compared, and the appropriate diameter was determined through this preliminary analysis.

Since the assumed defect is very small compared with clasp, a classical zooming $\mathrm{FEM}^{29,30)}$ was adopted to model the defect. This method uses global and local models, and local problem is solved after solving the global problem by applying the displacements obtained by the global analysis at the boundary of local model. In Fig. 4(a), both global and local models for zooming FEM are shown. The red colored line in the left figure shows the region of the local model in $z$-x plane. The thickness of local model, $0.6 \mathrm{~mm}$, is thinner than that of global model, $4 \mathrm{~mm}$, in order to reduce the number of elements in the local model. The spherical defect exists in the center of local model. In this modeling, the assumed spherical defect is included only in the local model where stress concentration was observed. In the zooming FEM, the displacement on the boundary surface of the local model was calculated by interpolating the global displacement using the shape function of FEM. Hence, a Dirichlet problem is solved for the local model. In this analysis, quadratic 10 node tetrahedral elements were used. The numbers of elements are 26,870 for global model and 236,634 for local model when the diameter is $1 \mu \mathrm{m}$. Note that the thickness of the local model is thinner than that of the component. The zooming elasto-plastic analysis procedure was built up in the commercial code COMSOL Multiphysics ver.5.4. Together with the uncertainty in the probably initial defect, the influence of the uncertainty in the material properties on the fatigue life was investigated for the S-shaped component.

Through this numerical preliminary work for
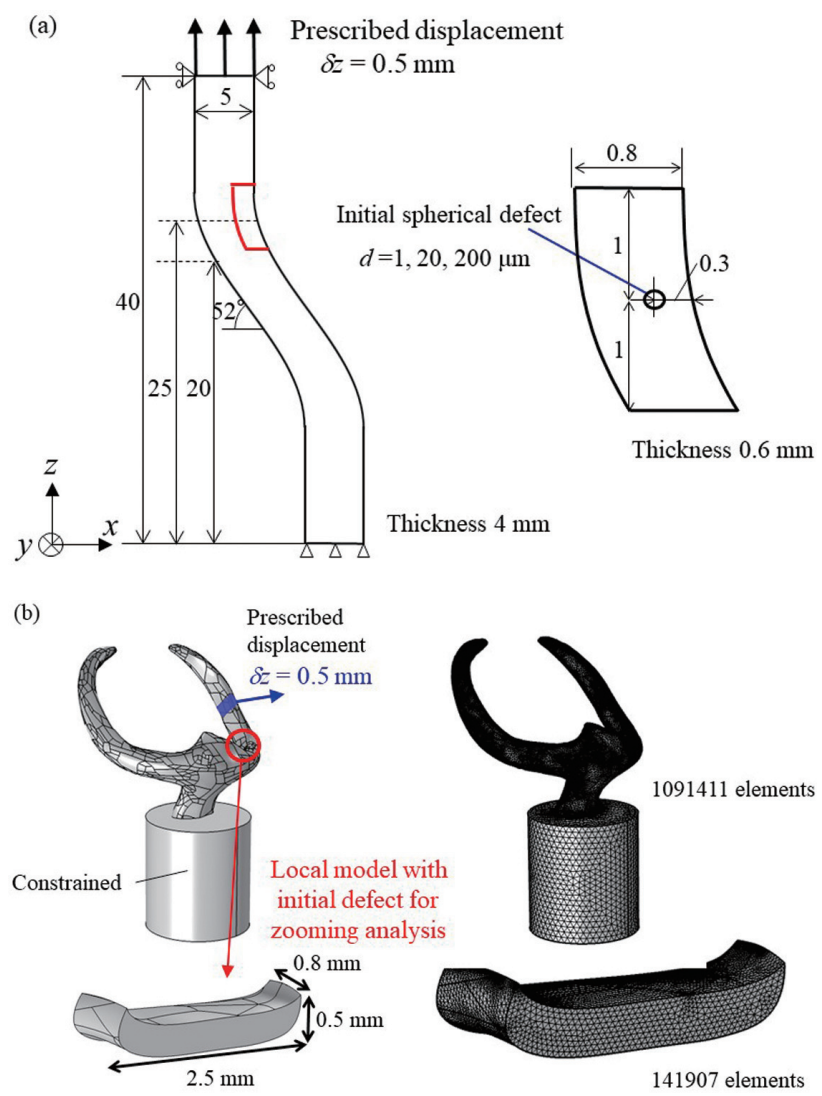

Fig. 4 Numerical models.

(a) Problem setup of S-shaped demonstrative component for zooming FEM considering three initial spherical defect models with diameters 1, 20 and $200 \mu \mathrm{m}$. The red colored line in the left figure shows the region of the local model in $z-x$ plane. The thickness of local model, $0.6 \mathrm{~mm}$, is thinner than that of global model, $4 \mathrm{~mm}$. The constraint condition and prescribed displacement up to 0.5 mm in FEA are shown. (b) Solid model and finite element mesh of global and local models of clasp with initial spherical defect of $1 \mu \mathrm{m}$ diameter in the center of local model. The constraint condition and prescribed displacement up to $0.5 \mathrm{~mm}$ in FEA are shown.

uncertainty quantification as described later, the spherical defect with $1 \mu \mathrm{m}$ diameter is assumed also in the clasp model in Fig. 4(b) as the probable defect in the region where stress concentration is observed. In Fig. 4(b), the left figure shows the solid global and local models for zooming FEM, and the right shows finite element mesh. The local model is embedded inside of the arm part of the clasp, where stress concentration was observed by the preliminarily analyzed linear elastic problem $^{31}$. The spherical defect is located in the center of the local model. The prescribed displacement up to 0.5 $\mathrm{mm}$ was applied in FEA in the measured direction in the fatigue test in Fig. 2(c) after the alignment of the clasp specimen using the jigs in Fig. 2(b). The cylindrical chuck 
part was fully fixed. In the experiment to compare the fatigue life with the numerical probabilistic prediction, approximately $0.25 \mathrm{~mm}$ displacement was applied in the same way with the numerical model.

\section{RESULTS}

Influence of diameter of initial defect

The numerical results of an S-shaped component without and with assumed initial spherical defects of 1,20 and $200 \mu \mathrm{m}$ diameters are described first. The distribution of maximum principal strain in the demonstrative S-shaped component is shown in Fig. 5(a). The left figure shows the result of global analysis, and the right figures show the results of local analyses when the diameter of spherical defects are $1 \mu \mathrm{m}$ and $200 \mu \mathrm{m}$. As explained in Fig. 4(a), the assumed initial defect was embedded in the region of the strain concentration indicated by a box in the left figure. Much higher strain was observed near the initial defect. Based on the proposed method to predict the fatigue life, the calculated fatigue lives are plotted in Fig. 5(b). This uncertainty quantification revealed that the initial defect gave serious influence

(a)

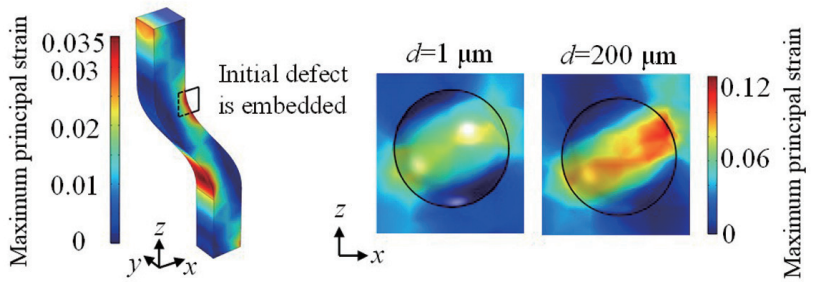

(b)

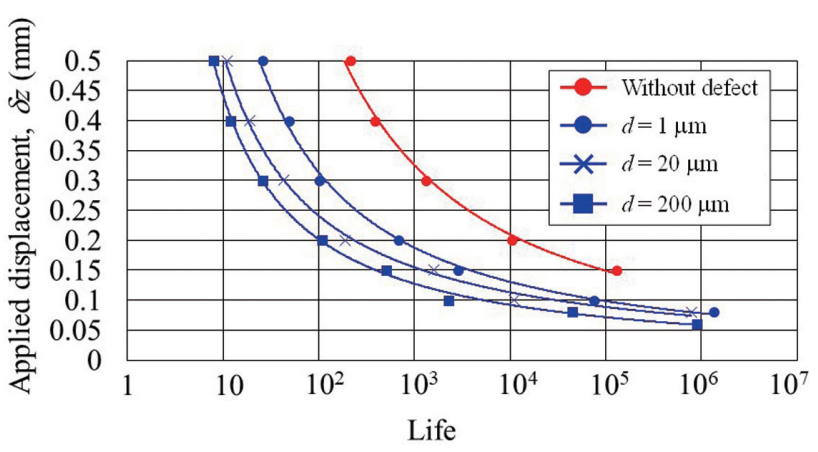

Fig. 5 Numerical results of S-shaped component.

(a) Maximum principal strain distribution for S-shaped component and magnified view near initial spherical defect that was embedded in the region of the strain concentration indicated by a box in the left figure. The left figure shows the result of global analysis, and the right figures show the results of local analyses when the diameters of spherical defects are $1 \mu \mathrm{m}$ and $200 \mu \mathrm{m}$. (b) Predicted fatigue life of S-shaped component without (red curve) and with 3 types of initial defect (blue curves). on the fatigue life even in the semi-logarithmic plot. It was supposed that $200 \mu \mathrm{m}$ and $20 \mu \mathrm{m}$ diameter defects are too large to express the variability in fatigue life. Hereafter, the diameter of the assumed initial defect was fixed to be $1 \mu \mathrm{m}$. The fatigue life predicted by the assumed $1 \mu \mathrm{m}$ diameter defect showed approximately $10^{-1}$ times shorter than the prediction without defect.

\section{Influence of variability in material properties}

Figure 6(a) shows the static response to the same problem setup in Fig. 4(a). We can observe clear differences among 3 material models. However, the fatigue lives in Fig. 6(b) show negligibly small differences in the semi-logarithmic plot. This result implies that the uncertainty in the material properties is less influential on the fatigue behaviors, but the influence of the initial defect on them is significant. To this end, in the analysis of the clasp, an average material model and $1 \mu \mathrm{m}$ initial defect were used.

Predicted fatigue life of clasp and comparison with experiment

The calculated maximum principal strain distribution

(a)

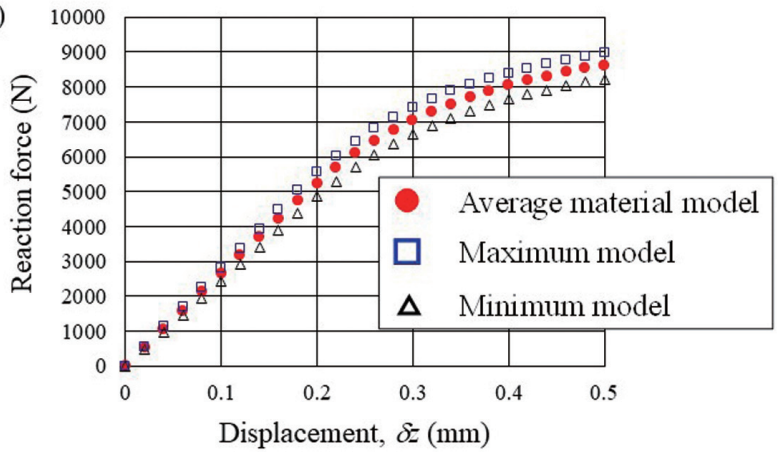

(b)

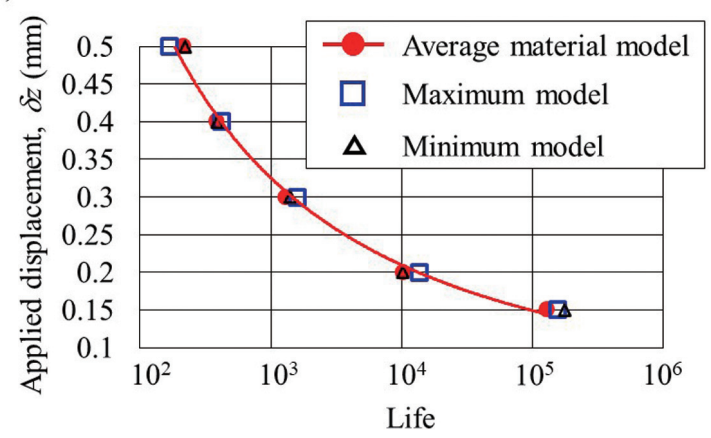

Fig. 6 Numerical results of the influence of variability in material properties on (a) static response and (b) fatigue life for S-shaped component when 0.15 to $0.5 \mathrm{~mm}$ prescribed displacements were applied as shown in Fig. 4(a).

In the fatigue life prediction in (b), the initial defect was not considered, but only the influence of material properties was analyzed. 
and the comparison with experimentally fractured position are shown in Fig. 7(a). The strain near the assumed initial spherical defect was higher than that in the global model. As in the graph of fatigue life in Fig. 7(b), 2 specimens among 3 were broken but 1 specimen had longer fatigue life than $1.5 \times 10^{5}$. The fractured positions in specimens No.1 and No.3 are compared with the calculated strain distribution as shown in Fig. 7(a). The fractured position lied almost in the local region indicated by blue line in Fig. 7(a) to embed an initial

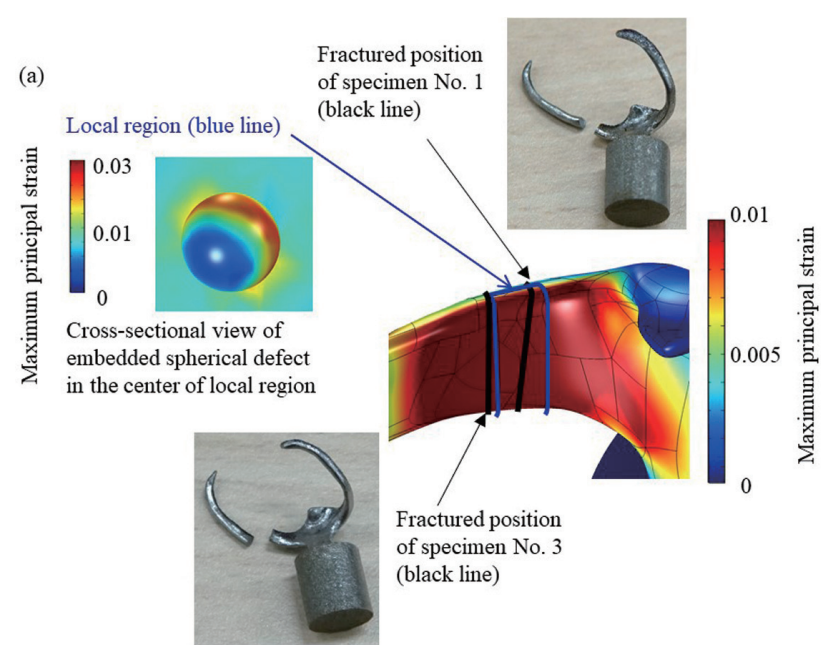

(b)

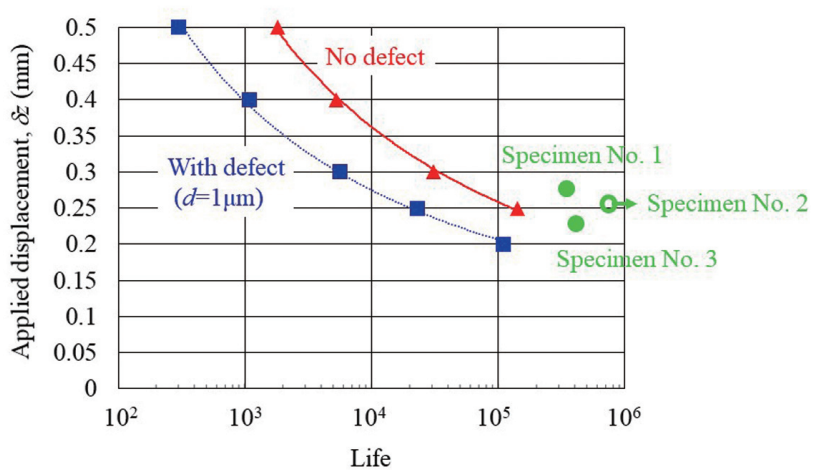

Fig. 7 Numerical results of probabilistic fatigue life prediction of clasp.

(a) Calculated maximum principal strain distribution in arm part. The fractured position in experiment was included in the local model. The maximum principal strains in the global model and at the cross-section near spherical defect in the local model are shown. (b) Numerically predicted fatigue life and comparison with experimental results of 3 specimens. The red curve is the numerical result without defect. The blue curve is the lower bound by assuming spherical defect of $1 \mu \mathrm{m}$ diameter as the most influential uncertainty factor. 0.2 to $0.5 \mathrm{~mm}$ prescribed displacements were applied in FEA, and approximately $0.25 \mathrm{~mm}$ stroke was given in experiment. defect with $1 \mu \mathrm{m}$ diameter in probabilistic FEA. Note that the initial defect is located in the center position of the local volume. In specimen No. 1, the fractured position was at the middle of the local region, which could support the proposed computational method.

In Fig. 7(b), the experimental results almost agree with the red line in the simulation, although extrapolation was not applied to the numerical results. The experimentally obtained fatigue lives of specimens No. 1 and No. 3 are almost the same, and the difference in applied stroke between them was almost the same with the numerically predicted variation at the life $N_{f}=10^{5}$. The variability range of the predicted fatigue life was smaller than the demonstrative S-shaped component, because more severe stress concentration in the global analysis was observed even without defect as shown in Fig. 7(a). Overall, the numerical prediction, especially the lower bound in blue line, underestimated the fatigue life compared with experimental facts, which is the safety side in the design.

\section{DISCUSSION}

The proposed numerical model to predict the variability in fatigue life was partially supported by the limited number of experimental results. However, the reason why the numerical prediction underestimated the fatigue life should be studied further by increasing the number of fatigue tests.

First, the values of applied displacement and force are discussed. If the numerical prediction including initial defect was used in the design of clasp, approximately $0.27 \mathrm{~mm}$ displacement was acceptable to satisfy the requirement of $10^{4}$ fatigue life, when 3 times attachment and detachment per day were supposed for 10 years. Therefore, the applied stroke of about $0.25 \mathrm{~mm}$ was given in the experiment. The experiments in many studies on the fatigue behaviors are force controlled ${ }^{18)}$. Marie et al. ${ }^{21)}$ measured clasp tip distortions as well as the force, and $0.105 \mathrm{~mm}$ to $0.319 \mathrm{~mm}$ displacement was measured. The applied displacement in our study is within the range of the above experiment. However, the standard deviation was very large ${ }^{21)}$. For instance, the distortion was $0.319 \pm 0.195 \mathrm{~mm}$. As the reason of the variability in experiments, an uncertainty factor is included especially in the constraint condition of the specimen. In our present study, the cylindrical chucking part was additively manufactured integrated with the clasp in this study. As the preliminary test, 9 specimens shown in Figs. 1(a), (b) were prepared by embedding the clasp body in resin and compared the static behaviors with 5 metal chucking specimens shown in Figs. 1(c), (d). The static load and stroke curves agreed perfectly with each other. It implied that the influence of both constraint condition and the building direction on the mechanical behaviors was negligible. But, the influence of the building direction should be studied furthermore, because Bayati et al. ${ }^{32}$ ) showed that the static and fatigue behaviors of the dumbbell type specimens placed in parallel to the powder bed and built in the thickness 
direction in AM showed larger variability. One of the merit to use the numerical simulation as the virtual test for design as well as for the quality assurance is that ideal constraint condition can be given to the specimen. The reason why larger displacements were not applied in our study lies in the limitation of the apparatus with the load cell capable of up to $50 \mathrm{~N}$. The reaction force in our experimental tests ranged between $36 \mathrm{~N}$ to $45 \mathrm{~N}$, which agrees with the numerical analyses by Sandu et al. ${ }^{17)}$. Concerning the fatigue life for clinical use, $10^{4}$ cycles is necessary if the clasp is used for 10 years with 3 times of attachment/detachment per day. Cheng et al..$^{33)}$ reported that, in their bending fatigue test of cobalt chromium alloy clasp, the retentive force decreased significantly after $0.72 \times 10^{4}$ cycles. Although the experimentally obtained fatigue lives by 3 specimens exceeded $10^{5}$, which satisfied the clinical requirement, the increase of fatigue tests can improve the reliability and validity of the proposed numerical model with $1 \mu \mathrm{m}$ diameter spherical initial defect.

Another possible reason for the underestimation of fatigue life may be the necessity of calibration of coefficient factors in SWT method. In this paper, the Bäumel \& Seeger rule was applied without recalibration. It is known that the Bäumel \& Seeger rule can be applied to titanium alloy and aluminum alloy. As one of the preliminary studies, we have confirmed the successful application of SWT method with Bäumel \& Seeger rule to published experimental data ${ }^{34)}$ for both materials. However, the published data ${ }^{34)}$ were not for additively manufactured specimens. It is very easy to recalibrate the parameters to fit the experimental results. Since the values of $b$ and $c$ in Eqs. (1) and (2) are negative, the fatigue life becomes larger if they are closer to zero. Also, if the ultimate strength is larger, then Eqs. (1) and (2) tells us that the fatigue life becomes longer.

Hence, focusing on the material properties in Table 1 , the additively manufactured titanium alloy in this study is discussed through comparison with the EOS data sheet. The Young's modulus was almost the same, but the yield stress and ultimate strength were lower. The lower ultimate strength can be one of the reasons for the underestimation by Bäumel \& Seeger rule. Or the tensile test specimen should be reconsidered. Although JIS Z 2241(2011) specimen with much larger dimensions than clasp was used, thin cylindrical tensile specimen with the same cross-sectional area with the arm part of the clasp should be considered together with the modification of parameters. Note that the ultimate strength and yield stress are lower than those of additively manufactured cobalt chromium alloy ${ }^{35}$. They are dependent on the chemical composition, but lower yield stress will result in higher plastic strain, which may shorten the fatigue life. The Young's modulus that determines the elastic strain is approximately half of cobalt chromium alloy ${ }^{35,36)}$, which increases the total strain and will give influence on the fatigue life. Hence, further studies are needed for the fatigue life of cobalt chromium alloy clasp, where the parameter identification for cobalt chromium alloy based on the Bäumel \& Seeger rule is also strongly required for the fatigue estimation of clasp.

Next, the quality of AM is discussed from the viewpoint of density and surface roughness. The measured apparent density was $4.4 \pm 0.08 \mathrm{~kg} / \mathrm{m}^{3}$, which agreed very well with the EOS data. The arithmetical mean deviation of the assessed profile $\mathrm{Ra}$ for 5 tensile specimens ranged between $15 \mu \mathrm{m}$ to $28 \mu \mathrm{m}$, and the maximum peak to valley height of the profile $\mathrm{Rz}$ ranged between $160 \mu \mathrm{m}$ to $229 \mu \mathrm{m}$. Ra was doubly larger than the EOS data, and Rz was much larger. As in the review paper ${ }^{11}$, the surface roughness has been a matter of concern in the dental studies on AM. In our study, as manufactured specimens were used and the influence of the surface roughness on the fatigue life was not investigated, which is one of the future works.

The geometrical accuracy of the additively manufactured clasp was proved by comparison with casting and milling ${ }^{2}$ as described in the uncertainty modeling. Takahashi et al. ${ }^{37)}$ also investigated the gap distances of additively manufactured titanium and/or titanium alloy clasps and tooth. They were under 100 $\mu \mathrm{m}$ and acceptable for clinical use from the viewpoint of fitness accuracy. Yager et al. ${ }^{4)}$ compared the static bending strength between casting and AM. The results showed almost the same mean values but the variability was much larger in the case of AM than casting. It is easily supposed that the fatigue lives are also largely scattered for additively manufactured clasp. This study is the first attempt to predict the variability by probabilistic FEA, as far as the authors know. One of the authors $^{38)}$ has ever proposed a probabilistic FEA based on more theoretical background for static analysis of oral implant surgery, but this paper proposed a new and practical method by analyzing only two static problems without and with initial defect for the quality assurance of patient-dependent clasp design. The proposed method does not explain the real phenomena and background physics of the fatigue behaviors. However, it is a simple and easy-to-use method for quick estimation before manufacturing, and was partially supported by fatigue test. In the experiment, final fracture occurred at the position where stress concentration was seen by FEA. Such agreement between FEA and experimental result has also been reported ${ }^{39}$. The authors ${ }^{39)}$ concluded that the stress distribution must be considered in the design of cast clasps to minimize fatigue failures. Our study gave the same result in the case of AM, and moreover, it has been revealed that probable AM process-induced defect $^{7,14,15,25)}$ can be very much influential on the fatigue life. In the future work, the microstructure observation of the fractured surface by SEM is required.

FEM has been used also for comparison between dissimilar materials ${ }^{40}$. However, most of the numerical studies analyzed static problems, and only a few studies $^{17)}$ are found that solved dynamic problems. By further studies on the validation by increasing the number of fatigue tests and the re-calibration of parameters for titanium allow as well as other materials, the probabilistic lower bound prediction will become a practical and effective tool for the design of AM clasps 
with respect to the fatigue life, because the variability must be considered for AM clasps due to many reasons such as defects to assure the long enough fatigue life. Also, the increase of static material tests is required to increase the credibility in the prediction of bounds in Fig. 6(b) for an S-shaped specimen. The influence of the variability in material properties on the fatigue life of clasp should be analyzed in the near future.

\section{CONCLUSION}

Within the limitations of this study, a probabilistic FEA procedure to predict the large variability in fatigue life has been proposed to be used as a virtual test in the design phase before manufacturing for quality assurance. The assumed very small initial spherical defect was modeled by zooming FEM. Limited number of experimental results could support partially the numerical results, especially in the comparison of fractured location. This paper discussed the clasp made of titanium alloy by $\mathrm{AM}$, but the application to cobalt chromium alloy and the extension and modification of the SWT method and Bäumel \& Seeger rule are expected.

\section{ACKNOWLEDGMENTS}

This study has been supported by JSPS Grants in Aid for Scientific Research B (20H02034). The authors would like to acknowledge the help of former students of Keio University, Mr. Shohei SATO in the numerical analyses and Mr. Tatsuto NOSE and Mr. Yutaro ABE in the experimental setup and in the study of uncertainty in the boundary conditions. There is no conflict of interest related to this study.

\section{REFERENCES}

1) Suzuki Y, Shimizu S, Waki T, Shimpo H, Ohkubo C. Laboratory efficiency of additive manufacturing for removable denture frameworks: A literature-based review. Dent Mater J 2021; 40: 265-271.

2) Tasaka A, Kato Y, Odaka K, Matsunaga S, Goto TK, Abe $\mathrm{S}$, et al. Accuracy of clasps fabricated with three different CAD/CAM technologies: Casting, milling and selective laser sintering. Int J Prosthodont 2019; 32: 526-529.

3) Chen RK, Jin Y, Wensman J, Shih A. Additive manufacturing of custom orthoses and prostheses -A review. Addit Manuf 2016; 12: 77-89.

4) Yager S, Ma J, Ozcan H, Kilinc HJ, Elwany AH, Karaman I. Mechanical properties and microstructure of removable partial denture clasps manufactured using selective laser melting. Addit Manuf 2015; 8: 117-123.

5) Culmone C, Smit G, Breedveld P. Additive manufacturing of medical instruments: A state-of-the-art review. Addit Manuf 2019; 27: 461-473.

6) Davenport JC, Basker RM,Heath JR, Ralph JP, Glantz PO, Hammond P. Clasp design. British Dent J 2001; 190: 71-81.

7) Kyogoku H, Ikeshoji TT. A review of metal additive manufacturing technologies: Mechanism of defects formation and simulation of melting and solidification phenomena in laser powder bed fusion process. Mech Eng Rev 2019; 7: $1-19$.

8) Leary M, Merli L, Torti F, Mazur M, Brandt M. Optimal topology for additive manufacture: A method for enabling additive manufacture of support-free structures. Mater Des 2014; 63: 678-690.

9) Gaynor AT, Guest JK. Topology optimization considering overhang constraints: Eliminating sacrificial support material in additive manufacturing through design. Struct Multidisc Optim 2016; 54: 1157-1172.

10) Takano N,Zako M. Integrated design of graded microstructures of heterogeneous materials. Arch Appl Mech 2000; 70: 585596.

11) Mahbod M, Asgari M. Elastic and plastic characterization of a new developed additively manufactured functionally graded porous lattice structure: Analytical and numerical models. Int J Mech Sci 2019; 155: 248-266.

12) Zhou H, Zhao M, Ma Z, Zhang DZ, Fu G. Sheet and network based functionally graded lattice structures manufactured by selective laser melting: Design, mechanical properties, and simulation. Int J Mech Sci 2020; 175: 105480.

13) Takano N, Takizawa H, Wen P, Odaka K, Matsunaga S, Abe S. Stochastic prediction of apparent compressive stiffness of selective laser sintered lattice structure with geometrical imperfection and uncertainty in material property. Int $\mathrm{J}$ Mech Sci 2017; 134: 347-356.

14) Snow Z, Nassar AR, Reutzel EW. Invited review article: Review on the formation and impact of flaws in powder bed fusion additive manufacturing. Addit Manuf 2020; 36: 101457.

15) Akita M, Uematsu Y, Kakiuchi T, Nakajima M, Kawaguchi R. Defect-dominated fatigue behavior in type 630 stainless steel fabricated by selective laser melting. Mater Sci Eng A 2016; 666: 19-26.

16) Douellow C, Balandraud X, Duc E, Verquin B, Lefebvre F, Sar F. Rapid characterization of the fatigue limit of additivemanufactured maraging steels using infrared measurements. Addit Manuf 2020; 35: 101310.

17) Sandu L, Faur N, Bortun C. Finite element stress analysis and fatigue behavior of cast circumferential clasps. J Prosthet Dent 2007; 97: 39-44.

18) Kato Y, Tasaka A, Kato M, Wadachi J, Takemoto S, Yamashita S. Effects of repetitive insertion/removal cycles and simulated occlusal loads on retention of denture retainers. Dent Mater J 2021; 40: 1277-1283.

19) Behr M, Zeman F, Passauer T, Koller M, Hahnel S, Buergers $\mathrm{R}$, et al. Clinical performance of cast clasp-retained removable partial dentures: A retrospective study. Int J Prosthodont 2012; 25: 138-144.

20) Urano S, Hotta Y, Miyazaki T, Baba K. Bending properties of Ce-TZP/A nanocomposite clasps for removable partial dentures. Int J Proshodont 2015; 28: 191-197.

21) Marie A, Keeling A, Hyde TP, Nattress BR, Pavitt S, Murphy RJ, et al. Deformation and retentive force following in vitro cyclic fatigue of cobalt-chrome and aryl ketone polymer (AKP) clasps. Dent Mater 2019; 35: eII3-eI2I.

22) The American Society of Mechanical Engineers. Guide for Verification and Validation in Computational Solid Mechanics. ASME V\&V10-2006 (2006).

23) Ghosh S, Mahmoudi M, Johnson L, Elwany A, Arroyave R, Allaire D. Uncertainty analysis of microsegregation during laser power bed fusion. Modelling Simul Mater Sci Eng 2019; 27: 034002 .

24) Moussa A, Melancon D, El Elmi A, Pasini D. Topology optimization of imperfect lattice materials built with processinduced defects via powder bed fusion. Addit Manuf 2021; 37: 101608.

25) Kakiuchi T, Kawaguchi R, Nakajima M, Fujimoto K, Uematsu Y. Prediction of fatigue limit in additively manufactured Ti$6 \mathrm{Al}-4 \mathrm{~V}$ alloy at elevated temperature. Int J Fatigue 2019; 126: 55-61.

26) Smith KN, Watson P, Topper TH. A stress-strain function for the fatigue of metals, J Mater 1970; 5: 767-778. 
27) Bäumel A, Seeger T. Material Data for Cyclic Loading, Supplement 1, Elsevier Science Publishers, 1990.

28) Liao D, Zhu SP, Keshtegar B, Qian G, Wang Q. Probabilistic framework for fatigue life assessment of notched components under size effects. Int J Mech Sci 2020; 181: 105685.

29) Hirai I, Wang BP, Pilkey WD. An efficient zooming method for finite element analysis. Int J Numer Methods Eng 1984; 20: $1671-1683$.

30) Takano N, Uetsuji Y, Asai M. Micro Mechanical Simulation. Tokyo: Corona Publishing; 2008. p. 59. (In Japanese)

31) Takano N. Study on uncertainty factors for metal additive manufacturing by selective laser melting for stochastic finite element modelling. 14th World Congress on Computational Mechanics (WCCM-ECCOMAS Virtual Congress); 2021 Jan 11-15; Paris, France.

32) Bayati P, Jahadakbar A, Barati M, Nematollahi M, SaintSulprice L, Haghshenas M, et al. Toward low and high cycle fatigue behavior of SLM-fabricated NiTi: Considering the effect of build orientation and employing a self-heating approach. Int J Mech Sci 2020; 185: 105878.

33) Cheng H, Xu M, Zhang H, Wu W, Zheng M, Li X. Cyclic fatigue properties of cobalt-chromium alloy clasps for partial removable dental prostheses. J Prosthet Dent 2010; 104: 389396.
34) Carrion PE, Shamsaei N, Daniewicz SR, Moser RD. Fatigue behavior of Ti-6Al-4V ELI including mean stress effects. Int J Fatigue 2017; 99: 87-100.

35) Shirasu K, Wakabayashi N, Yoneyama T, Igarashi Y. Nonlinear finite element stress analysis of plastic deformation in Co-Cr wrought-wire clasps. Dent Mater 2008; 24: 1518-1524.

36) $\mathrm{Al}$ Jabbari YS. Physico-mechanical properties and prosthodontic applications of $\mathrm{Co}-\mathrm{Cr}$ dental alloys: A review of the literature. J Adv Prosthodont 2014; 6: 138-145.

37) Takahashi K, Torii M, Nakata T, Kawamura N, Shimpo H, Ohkubo C. Fitness accuracy and retentive forces of additive manufactured titanium clasp. J Prosthodont Res 2020; 64: 468-477.

38) Tawara D, Nagahata M, Takano N, Kinoshita H, Abe S. Probabilistic analysis of mechanical behavior of mandibular trabecular bone using a calibrated stochastic homogenization model. Acta Mech 2015; 10: 3275-3287.

39) Mahmoud A, Wakabayashi N, Takahashi H, Ohyama T. Deflection fatigue of TI-6Al-7Nb, Co-Cr, and gold alloy cast clasps. J Prost Dent 2005; 93: 183-188.

40) Reddy JC, Chintapatla SB, Srikakula NK, Juturu RKR, Paidi SK, Tedlapu SK, et al. Comparison of retention of clasps made of different materials using three-dimensional finite element analysis. J Clin Diagnostic Res 2016; 10: 13-16. 